\title{
O câncer de mama no estado do Pará, Brasil, e o papel da Liga Acadêmica de Oncologia na promoção da saúde feminina: um relato de experiência
}

\author{
Breast cancer in the state of Pará, Brazil, and the role of Liga Acadêmica \\ de Oncologia on the promotion of women health: an experience report
}

Tiago Galan de França ${ }^{1,2}$, Luis Eduardo Werneck de Carvalho ${ }^{2,3}$

1Departamento de Ciências Biológicas e da Saúde, Universidade do Estado do Pará (UEPA) - Belém (PA), Brasil.

${ }^{2}$ Oncológica do Brasil Ensino e Pesquisa (OBEP) - Belém (PA), Brasil.

${ }^{3}$ Programa de Pós-graduação em Ciência da Saúde, Faculdade de Medicina do ABC (FMABC) - Santo André (SP), Brasil.

DOI: http://dx.doi.org/10.7322/abcshs.v42i3.924

\section{RESUMO}

Introdução: O câncer de mama é um grande agressor da saúde feminina, além de ser o segundo câncer mais incidente nessa população. É uma doença geralmente insidiosa, acometendo mulheres em idade mais avançada. Quando presente em mulheres mais jovens, tem pior prognóstico. Assim, diagnóstico e tratamento precoces são essenciais para o bom prognóstico das pacientes. O objetivo deste estudo foi fazer um relato de experiência sobre uma ação de extensão realizada pela Liga Acadêmica de Oncologia do Pará (LAOPA) para detecção do câncer de mama em uma população de risco e discutir a importância de ações de extensão no Pará. Relato de experiência: Um mutirão de rastreio do câncer de mama foi realizado no município de Ananindeua, Pará, em outubro de 2015. Uma capacitação para os acadêmicos foi feita por meio de aulas expositivas sobre câncer de mama e um treino prático foi realizado em modelos anatômicos. A ação foi dividida em espaços, alguns dos quais destinavam-se às consultas e outro, a uma palestra com o tema "desmistificando o câncer de mama". Conclusão: $O 5^{\circ}$ Mutirão da Mama se mostrou extremamente importante para o contexto da saúde feminina no Pará, pois ofertou atendimento clínico e exames gratuitos a uma população de risco para a neoplasia de mama, tornando-se também um projeto símbolo da LAOPA.

Palavras-chave: programas de rastreamento; neoplasias da mama; mamografia.

\begin{abstract}
Introduction: Breast cancer is a major female health aggressor and the second most common cancer in women. It is usually an insidious disease, affecting women at a later age. When present in younger women, prognosis is worse. Thus, early diagnosis and treatment are essential for a good prognosis. The objective of this study was to present an experience report about an extension action performed by Liga Acadêmica de Oncologia do Pará (LAOPA) to detect breast cancer in a population at risk and to discuss the importance of extension actions in the State of Pará, Brazil. Experience report: A breast cancer screening effort was conducted in the city of Ananindeua, in October 2015. The academics were trained in lectures on breast cancer and through practice with anatomical models. The project was divided by locales, some of which were destined to the consultations and other to a lecture on the topic "demystifying breast cancer". Conclusion: The $5^{\text {th }}$ Mutirão da Mama was extremely important for the female health context in Pará, since it offered clinical care and free examination tests to a population at risk for breast neoplasia, also becoming a symbol project of LAOPA.
\end{abstract}

Keywords: mass screening; breast neoplasms; mammography. 


\section{INTRODUÇÃO}

$O$ câncer de mama é a segunda neoplasia maligna mais incidente em mulheres no Brasil, atrás apenas do câncer de pele não melanoma ${ }^{1}$. É uma doença geralmente insidiosa, atingindo indivíduos em idade mais avançada ${ }^{2}$. Quando presente em mulheres mais jovens, tem pior prognóstico. A sintomatologia abriga um espectro amplo, cuja clínica varia de acordo com o indivíduo ${ }^{3}$.

$\mathrm{O}$ estigma do câncer de mama inclui o caráter fúnebre concedido à doença em si, além das consequências do tratamento dessa neoplasia, que muitas vezes pode levar a cirurgias mutiladoras ${ }^{4}$. Assim, o diagnóstico e o tratamento precoces são essenciais para o bom prognóstico das pacientes 5 .

Parte do processo de rastreamento do câncer de mama se dá pela mamografia ${ }^{6}$. O Ministério da Saúde preconiza que as mulheres entre 50 e 69 anos devem se submeter ao exame de forma bie$\mathrm{nal}^{1}$. Porém, ainda há discussão em relação à eficácia do Sistema Único de Saúde em implementar essa cobertura, uma vez que um número expressivo de pacientes recebe diagnóstico do câncer de mama em estágio avançado ${ }^{7}$.

Tendo em vista o problema exposto, a Liga Acadêmica do Pará (LAOPA), com apoio da Oncológica Brasil - Ensino e Pesquisa (OBEP), idealizou no estado o Mutirão da Mama. A liga é fundamentada no tripé "ensino, pesquisa e extensão", com ênfase em Oncologia, e tem como membros acadêmicos de Medicina de todos os semestres, orientados por um docente. O projeto do mutirão consiste no atendimento gratuito de mulheres em diferentes cidades do estado para o rastreio do câncer de mama ou de lesões precursoras. Além disso, também auxilia no aprimoramento da formação dos acadêmicos de Medicina, ao possibilitar-lhes a chance de atender as pacientes e aprender sobre cuidados primários em saúde. Desde sua criação, há cinco anos, já foram atendidas mulheres nos municípios de Capanema, Marituba, Ananindeua e Belém.

Entendendo a importância da ação realizada, este estudo teve o objetivo de apresentar um relato de experiência do $5^{\circ}$ Mutirão da Mama da LAOPA, realizado em outubro de 2015.

\section{RELATO DE EXPERIÊNCIA}

Foi realizado um mutirão para rastreio de câncer de mama no município de Ananindeua, no dia 31 de outubro de 2015, no hospital Anita Gerosa. Participaram os membros da LAOPA, com apoio da OBEP.

A distribuição de fichas para o agendamento das consultas foi feita por profissionais de Unidades de Saúde da Família situadas em Ananindeua, meses antes da execução do projeto. Assim, os agentes comunitários de saúde, em conformidade com os enfermeiros e médicos das unidades, identificaram as pacientes em maior necessidade de atendimento e forneceram as fichas. Apesar do número restrito de consultas previamente estabelecido (200), em razão da demanda espontânea no momento do mutirão, esse número foi muito maior.
Antes do mutirão, foi realizada a preparação dos acadêmicos de Medicina para o atendimento das mulheres. Essa foi feita por profissionais médicos e pelos membros mais experientes da liga, exemplificando um atendimento e exame clínico de mamas em modelos anatômicos, explicando acerca da ficha de coleta de dados e esclarecendo os questionamentos mais comuns das mulheres.

Em seguida, foi realizado o mutirão de rastreamento. Durante o dia de atendimento do mutirão, foi disponibilizado um espaço para o fornecimento de informações sobre o câncer de mama, auxiliando a desmistificar o tema. Esse esforço de elucidação foi realizado pelos próprios estudantes, por meio de palestras no momento de espera das consultas.

Ao todo, mais de 200 mulheres tiveram consultas gratuitas para rastreio de lesões indicativas de câncer de mama. Durante o exame clínico, foram realizados os seguintes procedimentos:

- anamnese (realizada por meio de uma ficha de coleta de dados, os quais incluíram: identificação, queixa e duração; antecedentes tocoginecológicos, familiares e pessoais; e histórico de câncer, pessoal e na família);

- exame físico (inspeção estática e dinâmica; palpação linfonodal; e expressão papilar).

Aquelas que apresentaram informações indicativas de maior investigação, como história familiar de primeiro grau de câncer de mama ou lesões sugestivas, foram encaminhadas para exames de mamografia gratuitos, agendados no local da consulta. A reavaliação das pacientes com os exames foi assegurada por meio de fichas de retorno fornecidas no momento da consulta.

\section{DISCUSSÃo}

\section{O desenvolvimento do mutirão}

O Programa Nacional de Controle do Câncer de Mama tem ênfase em três pilares de prevenção: detecção e tratamento do câncer de mama, e garantia de diagnóstico de lesões palpáveis; oferta de mamografias de rastreamento ao público-alvo; e qualificação da rede de atenção ${ }^{8}$. Essas ações são dependentes de financiamento e gestão das esferas federal, estadual e municipal de governo, sendo um dos grandes desafios de sua continuidade a manutenção dos mecanismos de regulação, de modo a possibilitar o repasse correto de investimentos? O Mutirão da Mama é uma iniciativa privada cujos pilares têm essa mesma ênfase, ao disponibilizar exames clínicos e de imagem para rastreamento do câncer de mama. Além disso, há um fator preventivo no mutirão, o qual será discutido posteriormente.

Sobre os impactos promovidos pela iniciativa, ao longo de cinco anos de atividade o Mutirão da Mama já forneceu atendimento médico de qualidade a mais de 2 mil mulheres no estado 
do Pará. Muitas delas tiveram lesões precursoras do câncer de mama detectadas durante o exame físico e foram encaminhadas para mamografia gratuita e imediata. Vale lembrar que, no Pará, esse serviço ainda não atingiu o nível de cobertura recomendado pela Organização Mundial da Saúde ${ }^{10}$, e a iniciativa privada para o fornecimento gratuito desse exame se mostra de extremo valor.

O processo de criação de uma boa relação entre os acadêmicos e médicos do mutirão e a população a ser atendida teve início muito antes da consulta. As salas de espera serviram para discussões sobre o câncer de mama, com atividades como "100 mitos sobre câncer de mama" e espaços abertos para questionamentos. Grande parte das dúvidas se relacionava à necessidade da realização de mamografia e ao seu papel no diagnóstico da neoplasia mamária ${ }^{11}$.

Outro assunto levantado com as pacientes era a mastectomia e suas consequências para a mulher, uma dúvida comum em virtude dos fatores social e sexual atribuídos às mamas ${ }^{12}$. Questões como escolaridade, estado civil e capacidade de realização de reconstrução mamária são importantes para entender por que algumas pacientes apresentam melhor autoestima após a mastectomia do que outras ${ }^{13}$. Muitas das pacientes presentes na ação nunca haviam se submetido a consulta para avaliação das mamas. Outras nem mesmo haviam passado por qualquer consulta específica de saúde feminina em toda a vida.

Observou-se também grande receio em relação ao diagnóstico do câncer. A visão estigmatizada da doença como uma sentença de morte ainda é muito presente no imaginário popular ${ }^{14}$. A falta de conhecimento sobre o assunto também se mostra um fator agravante para o diagnóstico e a percepção do câncer de mama ${ }^{15}$, evidenciando a necessidade de reavaliar a forma como o tema é tratado na Atenção Básica.

Assim, a divulgação de informações claras para o entendimento leigo acerca do câncer de mama é imprescindível, ao incluir a difusão de conhecimento como um fator protetor contra o aparecimento da doença ou seu diagnóstico em estágios mais avançados. Além da atenção primária, o atendimento de pacientes para o rastreio de lesões precursoras é essencial. Desse modo, o Mutirão da Mama não é uma ação imediatista, e sim um projeto cujos resultados poderão ser observados em longo prazo, ao unir educação em saúde, rastreio e diagnóstico em uma única ação.

\section{A experiência do $5^{\circ}$ Mutirão da Mama}

Para os participantes, a possibilidade de aliar a teoria aprendida nas escolas de Medicina com a prática no mutirão serviu para fixar melhor o conhecimento, além de permitir maior compreensão do funcionamento e do atendimento em um sistema público de saúde.

Concomitantemente ao aprendizado acadêmico e profissional, o crescimento pessoal também se fez presente, auxiliando na obtenção de uma visão mais humanizada das pacientes. $\mathrm{O}$ acolhimento correto e a propagação de informações foram essenciais para a boa aceitação por parte das mulheres.

Além disso, a ação mostrou uma realidade muitas vezes ignorada, por não haver enfoque adequado na saúde feminina no estado do Pará, especialmente nas regiões interioranas. Muitas pacientes relataram desconhecer cuidados com a saúde íntima ou medidas de autoconhecimento do corpo, como o autoexame das mamas.

A experiência com o $5^{\circ}$ Mutirão da Mama pode ser caracterizada como enriquecedora. O benefício mútuo se fez presente, seja no olhar de uma paciente, feliz pela sua primeira consulta para avaliação das mamas, seja nas expressões cansadas, mas exultantes, de alunos e alunas que se dedicaram a levar um pouco de conhecimento e saúde a uma população cuja situação não poderia expressar melhor a necessidade de ações extensivas.

\section{CONCLUSÃO}

Em conclusão, o $5^{\circ}$ Mutirão da Mama revelou-se importante para o contexto da saúde feminina no Pará e para a formação médica dos acadêmicos participantes, proporcionando contato com o paciente e aprimorando sua prática clínica. Além disso, introduziu o estudante no contexto dos cuidados primários, tendo como base a prevenção e a promoção da saúde. Assim, o projeto abre precedentes para o aprimoramento dos programas de rastreio precoce dessa neoplasia.

\section{AGRADECIMENTOS}

Agradecemos à LAOPA pelo excelente trabalho executado durante o $5^{\circ}$ Mutirão da Mama, em 2015, sem o qual este relato não seria possível.

\section{REFERÊNCIAS}

1. Brasil. Ministério da Saúde. Instituto Nacional de Câncer José Alencar Gomes da Silva. Diretrizes para a detecção precoce do câncer de mama no Brasil. Rio de Janeiro: INCA; 2015.

2. Brenner DR, Brockton NT, Kotsopoulos J, Cotterchio M, Boucher $\mathrm{BA}$, Courneya KS, et al. Breast cancer survival among young women: a review of the role of modifiable lifestyle factors. Cancer Causes Control. 2016;27(4):459-72

http://dx.doi.org/10.1007/s10552-016-0726-5

3. Marsicano AP, Soares CC, Pemper KCO, Silva JS, Zem PS, Borges BE, et al. Câncer de mama. Rev Curso Enferm. 2015;4. 
4. Szvarça RR, loshii SO, Carvalho DR, Sokoloski WF. Regras de associação temporal em cancer de mama. Rev Eng Tecnol. 2016;7(4):84-90.

5. World Health Organization. WHO position paper on mammography screening. Genebra: World Health Organization; 2014.

6. Oeffinger KC, Fontham ETH, Etzioni R, Herzig A, Michaelson JS, Shih YC, et al. Breast cancer screening for women at average risk: 2015 guideline update from the American Cancer Society. Jama. 2015;314(15):1599-614.

http://dx.doi.org/10.1001/jama.2015.12783

7. Brito C, Portela MC, Vasconcellos MTL. Assistência oncológica pelo SUS a mulheres com câncer de mama no Estado do Rio de Janeiro. Rev Saúde Pública. 2005;39(6):874-81. http://dx.doi.org/10.1590/S0034-89102005000600002

8. Silva RCF. Mamografia e rastreamento mamográfico: o debate da detecção precoce do câncer de mama contextualizado para a realidade brasileira. In: Teixeira L. Câncer de mama, câncer de colo de útero: conhecimentos, políticas e práticas. Rio de Janeiro: Outras Letras; 2015. p.165-203

9. Marques CAV, Gutiérrez MGR, Figueiredo EN. Políticas de saúde pública para o controle do câncer de mama no Brasil. Rev Enferm UERJ. 2015;23(2):272-8.

http://dx.doi.org/10.12957/reuerj.2015.13632
10. Freitas JA, Oliveira BGP, Ferreira HH, Santo SRE, Santos $R H$. Análise do índice de cobertura da mamografia em mulheres entre 50 e 69 anos, por nível de ensino, segundo unidade de federação. Rev Med UFC. 2016;56(1):14-7. http://dx.doi.org/10.20513/2447-6595.2016v56n1p14-17

11. Azevedo RL, Gerótica RMG, Sanches TP. A Importância da Mamografia no Diagnóstico Precoce do Câncer de Mama. UNILUS Ensino Pesq. 2016;13(30):251.

12. Duarte TP, Andrade AN. Enfrentando a mastectomia: análise dos relatos de mulheres mastectomizadas sobre questões ligadas à sexualidade. Estud Psicol. 2003;8(1):155-63. http://dx.doi.org/10.1590/S1413-294X2003000100017

13. Gomes NS, Silva SR. Avaliação da autoestima de mulheres submetidas à cirurgia oncológica mamária. Texto Contexto Enferm. 2013;22(3):509-16.

http://dx.doi.org/10.1590/S0104-07072013000200029

14. Gontijo IBR, Ferreira CB. Sentimentos de mulheres jovens frente ao diagnóstico de câncer de mama feminino. Ciênc Saúde. 2014;7(1):2-10. http://dx.doi.org/10.15448/1983-652X.2014.1.15488

15. Bushatsky M, Cabral LR, Cabral JR, Barros MBSC, Gomes BMR, Figueira Filho ASS. Educação em saúde: uma estratégia de intervenção frente ao câncer de mama. Ciênc Cuid Saúde. 2015;14(1):870-8. http://dx.doi.org/10.4025/cienccuidsaude.v14i1.23259 